\title{
The assessment of rheumatologic immune-related adverse events with immune checkpoint inhibitors
}

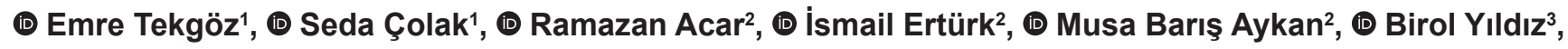 \\ (1) Nuri Karadurmuş ${ }^{2}$, (1) Muhammet Çınar ${ }^{1}$, (1) Sedat Yılmaz ${ }^{1}$
}

1 University of Health Sciences Turkey, Gülhane Faculty of Medicine, Department of Internal Medicine, Division of Rheumatology, Ankara, Turkey

2University of Health Sciences Turkey, Gülhane Faculty of Medicine, Department of Internal Medicine, Division of Oncology, Ankara, Turkey

${ }^{3}$ Elazig Medical Park Hospital, Clinic of Oncology, Elazig, Turkey

\section{Date submitted:}

26.06.2021

Date accepted:

23.11.2021

Online publication date: 15.03.2022

\section{Corresponding Author:}

Emre Tekgöz, M.D., University of Health Sciences Turkey, Gülhane Faculty of Medicine, Department of Internal Medicine, Division of Rheumatology, Ankara, Turkey dr.emretekgoz@hotmail.com

ORCID:

orcid.org/0000-0002-0866-1503

Keywords: Immune checkpoint inhibitors, adverse events, immunotherapy, inflammatory arthritis, polymyalgia rheumatica

\begin{abstract}
Aims: Immune-related adverse events (irAEs) may be observed due to the mechanism of action of immune checkpoint inhibitors (ICls). This study investigated the frequency of rheumatologic irAEs and characteristics of the patients who developed rheumatologic irAEs due to ICls.

Methods: This single-center, prospective, and observational study was conducted with the patients who received ICls due to malignancy between December 2018 and November 2019. The demographic characteristics, clinical characteristics, and inflammatory and noninflammatory irAEs were evaluated.

Results: The study included 38 patients (mean age: $54.5 \pm 19.2$ years, male: $65.8 \%$ ). Thirtyone $(86.1 \%)$ patients received nivolumab, $4(11.1 \%)$ patients received atezolizumab, and one (2.8\%) patient received pembrolizumab. The median number of treatment cycles with ICls was 11.5. Rheumatologic irAEs were observed in 20 (55.6\%) patients. Four (11.2\%) patients had inflammatory and 16 (44.5\%) patients had non-inflammatory rheumatologic irAEs. The median time from the initiation of $\mathrm{ICI}$ treatment to the development of rheumatologic irAEs was 3 months. All patients with inflammatory rheumatologic irAEs were on treatment with a programmed cell death protein 1 inhibitor. Prednisolone and methotrexate were the drugs used to improve inflammatory musculoskeletal symptoms.
\end{abstract}

Conclusions: Inflammatory rheumatological irAEs due to ICls were mostly polymyalgia rheumatica and rheumatoid arthritis-like symptoms. Low-dose corticosteroid therapy may be the appropriate choice of treatment of inflammatory rheumatologic irAEs.

\section{Introduction}

Antibodies against cytotoxic T-lymphocyte-associated protein-4 (CTLA-4), the programmed cell death protein 1 (PD1), and its ligand (PD-L1) that act as checkpoints in immune response pathways, are named as immune checkpoint inhibitors (ICls) and have begun used widely for treating hematologic malignancies, malignant melanoma, and solid cancers $(1,2)$. CTLA-4 on T cells binds CD80/86, and PD1 on T cells binds its ligands, PD-L1, and PD-L2, on antigen-presenting cells, which cause T cell inhibition (3). Immune checkpoint molecules provide self-regulation of immune tolerance, while these engagements also lead to cancer cells escaping from cytotoxic T-lymphocytes. As a result, cancer cell invasion becomes easier (4). ICls' anti-tumor effect emerges from the inhibition of the immune checkpoints that preserve the immunotolerance in $\mathrm{T}$ cells and the stimulation of the immune response of $T$ cells to tumor cells (5). However, due to their effects on immune response, a higher prevalence of immune-related adverse events (irAEs) may be observed due to uncontrolled $\mathrm{T}$ cell activation (1). 
Although irAEs may affect any organ system, cutaneous, gastrointestinal, pulmonary, and endocrine involvement may be more frequent. Rarely the patients may experience musculoskeletal, cardiovascular, hematologic, and neurologic irAEs $(6,7)$. IrAEs are usually low-grade, show a mild or moderate course, and regress without treatment; however, a multidisciplinary approach may be necessary for high-grade $\operatorname{irAEs}(1,7)$.

IrAEs are more frequent with PD1 and PD-L1 inhibitors compared with CTLA-4 inhibitors due to their actions in different steps of immune regulation (1). It was shown that rheumatologic irAEs are more common in patients who receive PD1 and PDL1 blockers or a combination of these agents compared with CTLA-4 inhibitors (8). This study evaluated the frequency of rheumatologic irAEs among patients who received $\mathrm{ICls}$ and the characteristics of these patients.

\section{Methods}

\section{Study design and sample}

This single-center, observational study with prospective enrollment included patients on treatment with PD1 and PD-L1 inhibitors. The participants were enrolled at Gülhane Training and Research Hospital, Ankara, Turkey, from December 2018 through November 2019. The inclusion criteria were (a) having received at least one dose of $\mathrm{ICl}$ due to malignancy, (b) no prior rheumatologic disease, (c) age 18 of age or older, and (d) agreeing to participate in the study. Exclusion criteria were pregnancy and breastfeeding. All patients provided written informed consent. The study protocol was approved by the Local Ethics Committee of Gülhane Training and Research Hospital (approval no: 18/282, November 21, 2018).

The treatment response to malignancy was evaluated by clinical, laboratory and radiological assessments. The development of rheumatologic and non-rheumatologic irAEs related to $\mathrm{ICl}$ use was evaluated.

\section{Evaluation of rheumatologic irAEs}

The patients were referred to the rheumatology outpatient clinic from the oncology outpatient clinic and evaluated by two rheumatologists for a history of rheumatologic disease, family history of rheumatologic disease, and new-onset rheumatologic symptoms. Clinical and demographic data were obtained from face-to-face interviews with patients and using the patient charts. Previously diagnosed rheumatologic diseases, family history of rheumatologic diseases, type of malignancy, duration of malignancy, $\mathrm{ICl}$ type, and the number of $\mathrm{ICl}$ cycles were recorded. If necessary, imaging methods (radiography, ultrasound, or magnetic resonance imaging) were performed in patients with rheumatologic irAEs. Depending on the type of manifestation, serum autoantibodies, human leukocyte antigen (HLA) B27, C-reactive protein, and erythrocyte sedimentation rate were measured. After the first assessment, the patients were re-evaluated for new-onset rheumatologic irAEs every 3 months for one year or in case of a musculoskeletal symptom.

Rheumatologic irAEs were determined as non-inflammatory and inflammatory manifestations. Arthralgia and myalgia without morning stiffness worsened with physical activity and lessened with rest, also, xerostomia, xerophthalmia, photosensitivity without proven connective tissue disease was considered noninflammatory rheumatologic adverse events. Inflammatory rheumatologic adverse events were defined as arthritis, bursitis, myositis, tenosynovitis, vasculitis, and connective tissue diseases and confirmed by examination, laboratory, and imaging methods $(3,9,10)$.

\section{Statistical Analysis}

All statistical analyses were performed using Statistical Package for the Social Sciences (SPSS) Statistics for Windows, version 11.5, SPSS Inc., Chicago. The normality assumption was assessed by the Kolmogorov-Smirnov test. Normally distributed continuous variables were expressed as mean \pm standard deviation, while non-normally distributed continuous variables were expressed as median (Q1-Q3). Categorical variables were summarized as counts (percentages).

\section{Results}

\section{Basic characteristics}

The study included 36 patients ( 25 male, $65.8 \%$ ) with a mean age of $54.5 \pm 19.2$ years. The median duration of malignancy was 26.5 (14-48) months. Eleven (30.6\%) patients had Hodgkin's lymphoma, $10(27.8 \%)$ patients had malign melanoma, 9 $(25.0 \%)$ patients had renal cell cancer, $4(11.1 \%)$ patients had small cell lung cancer, $1(2.8 \%)$ patient had non-small cell lung cancer, and 1 (2.8\%) hepatocellular cancer. Seventeen (47.2\%) patients had metastatic disease. Thirty-two (88.9\%) patients received PD1 inhibitors (nivolumab and pembrolizumab), and $4(11.1 \%)$ patients received PD-L1 inhibitors (atezolizumab). Nivolumab was used in $31(86.1 \%)$, atezolizumab in $4(11.1 \%)$ patients, and pembrolizumab in $1(2.8 \%)$ patient. The median number of $\mathrm{ICl}$ cycles was 11.5 (8-23). All patients received chemotherapy, and 12 (33.3\%) patients received radiotherapy before initiation of $\mathrm{ICl}$ therapy.

Ten $(27.8 \%)$ patients achieved complete response, 11 $(30.6 \%)$ patients had partial regression, and $8(22.2 \%)$ patients had stable disease with ICls. Progression was recorded in 7 $(19.4 \%)$ patients and $4(11.1 \%)$ patients who died during the follow-up period (Table 1).

\section{Rheumatologic evaluation}

Rheumatologic irAEs were recorded in 20 (55.6\%) patients. The median time to the occurrence of rheumatologic adverse events after initiation of $\mathrm{ICl}$ was 3 (1.5-7) months. The most 
common musculoskeletal manifestation was arthralgia in 15 $(41.7 \%)$ patients. The other irAEs were xerostomia in 5 (13.9\%) patients, xerophthalmia in $3(8.3 \%)$ patients, photosensitivity in $3(8.3 \%)$ patients, arthritis in $2(5.6 \%)$ patients, bursitis in 2 $(5.6 \%)$ patients, polymyalgia rheumatica (PMR) like syndrome in $2(5.6 \%)$ patients, tendinitis in $1(2.8 \%)$ patient and myalgia in $1(2.8 \%)$ patient (Table 2$)$. Among patients with inflammatory rheumatologic irAEs, $2(5.6 \%)$ patients had PMR-like symptoms, and $2(5.6 \%)$ patients had rheumatoid arthritis (RA) like symptoms. The mean time of the initiation of PMR-like symptoms was 2.5 months, and it was 4 months for RA-like symptoms. Sixteen $(44.5 \%)$ patients had non-inflammatory rheumatologic irAEs. All patients with inflammatory rheumatologic irAEs were onPD1 inhibitor treatment (Figure 1). Rheumatoid factor, anticyclic citrullinated peptide, antinuclear antibody, and HLA B27 were negative in all patients.

Arthralgia was common in the shoulders, elbows, and knees. Two patients had arthritis in the wrists. Bursitis in two patients was in the subacromial and subdeltoid bursa. Tenosynovitis of musculus extensor carpi ulnaris was recorded in two patients. Four $(11.1 \%)$ patients with arthritis, bursitis and tenosynovitis received prednisolone therapy, and one $(2.8 \%)$ patient who had corticosteroid-resistant arthritis received methotrexate. Prednisolone doses of $10 \mathrm{mg} /$ day were sufficient for healing musculoskeletal manifestations. Seven (19.4\%) patients with non-inflammatory rheumatologic irAE were treated with nonsteroid anti-inflammatory drugs. Ten (27.8\%) patients had non-rheumatologic irAEs (Table 2). The patients with xerophthalmia and xerostomia had no radiotherapy to the gland localization.

\begin{tabular}{|c|c|c|}
\hline \multicolumn{3}{|c|}{$\begin{array}{l}\text { Patients receving ICI } \\
n=36\end{array}$} \\
\hline \multicolumn{3}{|c|}{$\begin{array}{l}\text { Rhematologic irAEs } \\
n=20(55.6 \%)\end{array}$} \\
\hline $\begin{array}{c}\text { Inflan } \\
\mathrm{n}=4\end{array}$ & $\begin{array}{l}\text { atory } \\
1 \%)\end{array}$ & $\begin{array}{c}\text { Non-inflammatory } \\
n=16(44.5 \%)\end{array}$ \\
\hline $\begin{array}{l}\text { PMR patern } \\
n=2(5.6 \%)\end{array}$ & $\begin{array}{l}\text { RA patern } \\
\mathrm{n}=2(5.6 \%)\end{array}$ & \\
\hline $\begin{array}{c}\text { Nivolumab, } \mathrm{n}=1 \\
\text { Pembrolizumab, } \mathrm{n}=1\end{array}$ & Nivolumab, $n=2$ & $\begin{array}{l}\text { Nivolumab, } n=15 \\
\text { Atezolizumab, } n=1\end{array}$ \\
\hline Corticosteroids, $n=2$ & $\begin{array}{l}\text { Corticosteroids, } n=2 \\
\text { Methotrexate, } n=1\end{array}$ & $\begin{array}{c}\text { NSAID, } n=7 \\
\text { None, } n=8\end{array}$ \\
\hline
\end{tabular}

Figure 1. The immune-related rheumatologic adverse events of the study group

ICl: Immune checkpoint inhibitor, irAEs: Immune-related adverse events, RA: Rheumatoid arthritis, PMR: Polymyalgia rheumatica, NSAID: Non-steroidal antiinflammatory drug

\begin{tabular}{|c|c|c|}
\hline & & Patients $(n=36)$ \\
\hline Gender, n (\%) & Female & $11(30.6)$ \\
\hline Age, years, mean $\pm S D$ & & $54.5 \pm 19.2$ \\
\hline Disease duration for malignancies (months), median (Q1-Q3) & & $26.5(14-48)$ \\
\hline \multirow{4}{*}{ Types of malignancy, n (\%) } & Renal cell cancer & $9(25.0)$ \\
\hline & Small cell lung cancer & $4(11.1)$ \\
\hline & Non-small cell lung cancer & $1(2.8)$ \\
\hline & Hepatocellular cancer & $1(2.8)$ \\
\hline Type of $\mathrm{ICl}, \mathrm{n}(\%)$ & Pembrolizumab & $1(2.8)$ \\
\hline Number of ICl cycle, median (Q1-Q3) & & $11.5(8-23)$ \\
\hline Patients received previous chemotherapy, $\mathrm{n}(\%)$ & & $36(100)$ \\
\hline Patients received previous radiotherapy, n (\%) & & $12(33.3)$ \\
\hline \multirow{4}{*}{ Response of the malignancy, $\mathrm{n}(\%)$} & Complete response & $10(27.8)$ \\
\hline & Partial regression & $11(30.6)$ \\
\hline & Stable disease & $8(22.2)$ \\
\hline & Progression & 7 (19.4) \\
\hline
\end{tabular}




\begin{tabular}{|c|c|c|}
\hline & & Patients $(n=36)$ \\
\hline \multicolumn{2}{|c|}{ The time between initiation of ICI treatment and the occurrence of rheumatologic irAEs, months, median (Q1-Q3) } & $3(1.5-7)$ \\
\hline \multicolumn{2}{|c|}{ The number of patients with rheumatologic irAEs, $n(\%)$} & $20(55.6)$ \\
\hline \multirow{9}{*}{ Rheumatologic irAEs, $n(\%)$} & Arthralgia & $15(41.7)$ \\
\hline & Xerostomia & $5(13.9)$ \\
\hline & Xerophthalmia & $3(8.3)$ \\
\hline & Photosensitivity & $3(8.3)$ \\
\hline & Arthritis & $2(5.6)$ \\
\hline & Bursitis & $2(5.6)$ \\
\hline & Polymyalgia rheumatica like syndrome & $2(5.6)$ \\
\hline & Tendinitis & $1(2.8)$ \\
\hline & Myalgia & $1(2.8)$ \\
\hline \multicolumn{2}{|c|}{ The number of patients with non-rheumatologic irAEs, $n(\%)$} & $10(27.8)$ \\
\hline \multirow{5}{*}{ Non-rheumatologic irAEs, n (\%) } & Rash & $6(16.7)$ \\
\hline & Xerosis cutis, pruritus & $2(5.6)$ \\
\hline & Alopecia & $1(2.8)$ \\
\hline & Thyroiditis & $1(2.8)$ \\
\hline & Seborrheic dermatitis & $1(2.8)$ \\
\hline \multirow{3}{*}{ Treatment of the rheumatologic irAEs, $n(\%)$} & NSAID & $7(19.4)$ \\
\hline & Corticosteroid & $4(11.1)$ \\
\hline & Methotrexate & $1(2.8)$ \\
\hline
\end{tabular}

\section{Discussion}

ICls, different from classical chemotherapeutic agents, show their anti-tumor effect by activating the immune system and attain widespread benefit for treating oncology patients. ICls prevent the immune pathways that inhibit the autoactivation and overreaction of the immune system. Due to the activation mechanism of these drugs, irAEs may appear during the treatment $(1,2)$. According to the current study, which evaluated the rheumatologic irAEs in patients using ICls, the findings requiring treatment were particularly in patients who had similar symptoms with RA and PMR.

Monoclonal antibodies that blockade immune checkpoints to treat various types of malignancies in different stages are used widely. CTLA-4, PD1, and PD-L1 provide the balance between activation and inhibition of T cells (11). The main goal of ICls is to increase the immune response to tumor cells by inhibiting immune checkpoints that prevent the inhibition of cytotoxic T-lymphocytes (12). In the central lymphoid tissues, to inhibit $T$ cell activation, CTLA-4 prevents the engagement between CD80/86 on antigen-presenting cells and CD28 on T cells. Thus, the antibodies that inhibit CTLA-4 activate T cells, leading to an increase in peripheral T cell migration and attack on tumor cells. PD1 commonly exists in peripheral T cells. When PD1 binds to its ligands, PD-L1, and PD-L2, cancer cells proliferate due to T cell inhibition. The antibodies that inhibit PD1 and PD-L1 increase the
T cell response against cancer cells (13). The accomplishment of $\mathrm{ICls}$ in achieving remission or preventing disease progression in resistant or metastatic malignant melanoma, lung cancer, renal cell cancer, and Hodgkin's lymphoma provide their widespread use in oncology. In this study, ICls were used frequently in patients with Hodgkin's lymphoma, malignant melanoma, and renal cell cancer consistent with the recommendations.

However, inhibition of immune checkpoints with ICls with excessive activation of the immune system may lead to undesirable immune and inflammatory events. These irAE affect many organ systems and may lead to complications even may be fatal (13). The irAE rate with PD 1 and PD-L1 inhibitors was reported as $70 \%$, whereas it may be up to $95 \%$ among patients using combination therapy (PD 1, PD-L1, and CTLA 4) (14-16). Hofmann et al. (17) investigated the irAEs in 496 patients with malignant melanoma receiving nivolumab and pembrolizumab. According to their study, there were 242 irAEs in 138 patients in the skin, endocrine, renal and gastrointestinal systems, but no rheumatologic irAE existed. However, differing from this study, we found that rheumatologic irAEs were more frequent than other irAEs. The second most frequent irAE was related to the skin with a similar frequency of rash, pruritus, and alopecia to the literature (17).

The rheumatological side effects that may develop due to an $\mathrm{ICl}$ treatment have not been recorded in most studies 
so far and presented as case series. Arthralgia and myalgia can be considered paraneoplastic or related to conventional chemotherapeutic agents; oral dryness and ocular dryness can be attributed to radiotherapy, which may prevent the true frequency assessment regarding the rate of rheumatologic irAEs related to $\mathrm{ICls}$. The rate of rheumatologic irAEs was reported to be $1.5-22 \%$ in the literature $(2,18)$. The current study found a higher rate of rheumatologic irAEs than the previous studies in the literature. Higher rates of rheumatologic irAEs may be related to the assessment of the patients periodically during the study period by rheumatologists in terms of the presence of any rheumatologic symptoms.

Whereas arthralgia and myalgia are the most frequently reported adverse events due to $\mathrm{ICI}$, recent studies determined two groups of rheumatologic irAEs, PMR-like syndrome, and inflammatory arthritis. PMR-like syndrome and inflammatory arthritis are more frequent and comprise a higher rate among systemic symptoms $(3,10,19-21)$. According to a prospective study conducted by Kostine et al. (19) rheumatologic irAEs were characterized as inflammatory and non-inflammatory, and arthralgia was accepted as a non-inflammatory irAE. In the same study, inflammatory rheumatologic irAEs were frequently seen as PMR and RA patterns. A review showed that rheumatologic irAEs are usually detected as PMR-like symptoms, tenosynovitis, and arthritis of hand joints, and with decreasing frequency asymmetric oligoarthritis, reactive arthritis, and psoriatic arthritis (13). Inflammatory arthritis as RA and PMR-like patterns was frequent in the current study, especially among patients who received PD1 inhibitors. The association between PD1 and PD$L 1$ receptors affects the pathophysiology of RA and PMR. PD1 polymorphism is associated with increased susceptibility to RA. Also, soluble and transmembrane PD1 expression decreases in patients with RA $(22,23)$.

Rheumatologic irAEs such as inflammatory arthritis may occur as early as in the first month of the ICI therapy, while they may also arise after two years of treatment (13). According to the literature, PMR-like symptoms may develop earlier than RAlike symptoms related to $\mathrm{ICl}$ use (9). Similar to the literature, in the current study, we found that PMR-like symptoms have developed earlier than RA-like symptoms. According to the literature, autoantibody positivity was usually absent in patients with rheumatologic irAEs (9). Rheumatoid factor and anti-cyclic citrullinated peptide may be found positive in a few patients (24). A positive antinuclear antibody can rarely be found at low titers (9). No patient had a positive rheumatoid factor, anti-cyclic citrullinated peptide, or antinuclear antibody in the current study. Minor salivary gland biopsy was not performed in patients with sicca symptoms due to negative antinuclear antibodies and no other system findings associated with connective tissue disease. Recent studies showed that Sjogren's syndrome may develop during $\mathrm{ICl}$ therapy. However, in contrast with classical
Sjogren's syndrome, antinuclear, anti-Ro, and anti-La antibody positivity may be seen in rare cases, and pathological evaluation may show differences due to acinar ductal destruction caused by T-lymphocyte infiltration $(25,26)$.

The main concern about the management of inflammatory rheumatologic irAEs, especially using glucocorticoids, is the consideration of decreasing the effect of $\mathrm{ICl}$ treatment. Glucocorticoids may diminish the efficiency and strength of lymphocytes that infiltrate tumor tissue (27). Thus, high-dose glucocorticoid usage is recommended only for life-threatening organ manifestations. Besides, using $10 \mathrm{mg} /$ day prednisolone for up to six weeks was found safe and did not decrease the efficacy of the $\mathrm{ICl}$ treatment in patients receiving PD1 and PDL1 inhibitors $(9,28)$. European League Against Rheumatism (EULAR) recommended methotrexate as the corticosteroid tapering agent for patients with continuing symptoms under 10 $\mathrm{mg} /$ day prednisolone treatment $(9,29)$. According to the EULAR recommendations, we treated rheumatologic inflammatory irAEs with $10 \mathrm{mg} /$ day prednisolone. This treatment was sufficient for providing and maintaining remission. Only one patient received methotrexate due to ongoing symptoms despite corticosteroid therapy.

There are some limitations of the current study. First, our study had a small sample from a single center. Further studies with larger samples and longer follow-up times may provide more data regarding rheumatologic irAEs. Two patients with sicca symptoms had no autoantibodies for Sjogren's syndrome, therefore, a minor salivary gland biopsy had not been performed.

\section{Conclusion}

In conclusion, immune checkpoint receptors help balance the immune system by a downregulation mechanism to ensure while the immune system acts against foreign, non-self, and infectious agents. This mechanism is the essential protector mechanism for preventing the development of autoimmunity and autoimmunity-related diseases. The central aim of $\mathrm{ICl}$ therapy is to impede the immune inhibitory pathways for providing $T$ cell attacks against cancer cells that hide from the immune system. Consequently, the inhibition of immune checkpoints may cause rheumatologic irAEs. Physicians should be cautious about potential inflammatory rheumatologic irAEs that are not rare and can be overlooked or misdiagnosed, particularly the PMR-like and RA-like symptoms.

\section{Ethics}

Ethics Committee Approval: The study was approved by the Local Ethical Committee of Gülhane Training and Research Hospital with approval number $18 / 282$ on November $21^{\text {st }}$ of 2018.

Informed Consent: Consent form was filled out by all participants. 
Peer-review: Externally peer-reviewed.

\section{Authorship Contributions}

Surgical and Medical Practices: E.T., S.Ç., R.A., İ.E., M.B.A., B.Y., N.K., M.Ç., S.Y., Concept: E.T., S.Ç., M.Ç., S.Y., Design: E.T., S.Ç., M.Ç., S.Y., Data Collection or Processing: E.T., S.Ç., R.A., I.E., M.B.A., B.Y., N.K., M.Ç., S.Y., Analysis or Interpretation: E.T., S.Ç., R.A., İ.E., M.B.A., B.Y., N.K., M.Ç., S.Y., Literature Search: E.T., S.Ç., Writing: E.T., S.Ç., R.A., I.E., M.B.A., B.Y., N.K., M.Ç., S.Y.

Conflict of Interest: No conflict of interest was declared by the authors.

Financial Disclosure: The authors declared that this study received no financial support.

\section{References}

1. Postow MA, Sidlow R, Hellmann MD. Immune-Related Adverse Events Associated with Immune Checkpoint Blockade. N Engl J Med. 2018;378:158-168.

2. Le Burel S, Champiat S, Mateus C, et al. Prevalence of immune-related systemic adverse events in patients treated with anti-Programmed cell Death 1/anti-Programmed cell Death-Ligand 1 agents: A single-centre pharmacovigilance database analysis. Eur J Cancer. 2017;82:34-44.

3. Cappelli LC, Gutierrez AK, Bingham CO 3rd, Shah AA. Rheumatic and musculoskeletal immune-related adverse events due to immune checkpoint inhibitors: a systematic review of the literature. Arthritis Care Res (Hoboken). 2017;69:1751-1763.

4. Ghiotto M, Gauthier L, Serriari N, et al. PD-L1 and PD-L2 differ in their molecular mechanisms of interaction with PD-1. Int Immunol. 2010;22:651-660.

5. Van der Vlist M, Kuball J, Radstake TRD, Meyaard L. Immune checkpoints and rheumatic diseases: what can cancer immunotherapy teach us? Nat Rev Rheumatol. 2016;12:593604.

6. Jia $\mathrm{XH}$, Geng $\mathrm{LY}$, Jiang PP, et al. The biomarkers related to immune related adverse events caused by immune checkpoint inhibitors. J Exp Clin Cancer Res. 2020;39:284.

7. Weber JS, Hodi FS, Wolchok JD, et al. Safety Profile of Nivolumab Monotherapy: A Pooled Analysis of Patients With Advanced Melanoma. J Clin Oncol. 2017;35:785-792.

8. Khoja L, Day D, Wei-Wu Chen T, Siu LL, Hansen AR. Tumourand class-specific patterns of immune-related adverse events of immune checkpoint inhibitors: A systematic review. Ann Oncol. 2017;28:2377-2385.

9. Kostine M, Finckh A, Bingham CO, et al. EULAR points to consider for the diagnosis and management of rheumatic immune-related adverse events due to cancer immunotherapy with checkpoint inhibitors. Ann Rheum Dis. 2021;80:36-48.

10. Richter MD, Crowson C, Kottschade LA, Finnes HD, Markovic $\mathrm{SN}$, Thanarajasingam U. Rheumatic syndromes associated with immune checkpoint inhibitors: a Single-Center cohort of Sixty-One patients. Arthritis Rheumatol. 2019;71:468-475.
11. Postow MA, Callahan MK, Wolchok JD. Immune checkpoint blockade in cancer therapy. J Clin Oncol. 2015;33:1974-1982.

12. Dyck L, Mills KHG. Immune checkpoints and their inhibition in cancer and infectious diseases. Eur J Immunol. 2017;47:765779.

13. Jamal S, Hudson M, Fifi-Mah A, Ye C. Immune-related adverse events associated with cancer immunotherapy: a review for the practicing rheumatologist. J Rheumatol. 2020;47:166-175.

14. Topalian SL, Hodi FS, Brahmer JR, et al. Safety, activity, and immune correlates of anti-PD-1 antibody in cancer. $\mathrm{N}$ Engl J Med. 2012;366:2443-2454

15. Hodi FS, O'Day SJ, McDermott DF, et al. Improved survival with ipilimumab in patients with metastatic melanoma. $\mathrm{N}$ Engl J Med. 2010;363:711-723.

16. Brahmer JR, Tykodi SS, Chow LQM, et al. Safety and Activity of Anti-PD-L1 Antibody in Patients with Advanced Cancer. N Engl J Med. 2012;366:2455-2465.

17. Hofmann L, Forschner A, Loquai C, et al. Cutaneous, gastrointestinal, hepatic, endocrine, and renal side-effects of anti-PD-1 therapy. Eur J Cancer. 2016;60:190-209.

18. Betof AS, Nipp RD, Giobbie-Hurder A, et al. Impact of age on outcomes with immunotherapy for patients with melanoma. Oncologist. 2017;22:963-971.

19. Kostine M, Rouxel L, Barnetche T, et al. Rheumatic disorders associated with immune checkpoint inhibitors in patients with cancer-clinical aspects and relationship with tumour response: a single-centre prospective cohort study. Ann Rheum Dis. 2018;77:393-398.

20. Zong H, Zhou J, Xu D, Zeng X. Rheumatic immune-related adverse events induced by immune checkpoint inhibitors. Asia Pac J Clin Oncol. 2021;17:178-185.

21. Abdel-Rahman O, Eltobgy M, Oweira H, Giryes A, Tekbas A, Decker M. Immune-related musculoskeletal toxicities among cancer patients treated with immune checkpoint inhibitors: a systematic review. Immunotherapy. 2017;9:1175-1183.

22. Garel B, Kramkimel N, Trouvin AP, Frantz C, Dupin N. Pembrolizumab-induced polymyalgia rheumatica in two patients with metastatic melanoma. Joint Bone Spine. 2016;84:233-234.

23. Ceeraz S, Nowak EC, Burns CM, Noelle RJ. Immune checkpoint receptors in regulating immune reactivity in rheumatic disease. Arthritis Res Ther. 2014;16:469.

24. Belkhir R, Le Burel S, Dunogeant L, et al. Rheumatoid arthritis and polymyalgia rheumatica occurring after immune checkpoint inhibitor treatment. Ann Rheum Dis. 2017;76:17471750.

25. Brugués AO, Sibaud V, Herbault-Barrés B, et al. Sicca syndrome induced by immune checkpoint inhibitor therapy: optimal management still pending. Oncologist. 2020;25:391395.

26. Ramos-Casals M, Maria A, Suárez-Almazor ME, et al. Sicca/ Sjögren's syndrome triggered by PD-1/PD-L1 checkpoint inhibitors. Data from the International ImmunoCancer Registry (ICIR). Clin Exp Rheumatol. 2019;37(Suppl 118):114-122. 
27. Draghi A, Borch TH, Radic HD, et al. Differential effects of corticosteroids and anti-TNF on tumor-specific immune responses: implications for the management of irAEs. Int J Cancer. 2019;145:1408-1413.

28. Mitchell EL, Lau PKH, Khoo C, et al. Rheumatic immunerelated adverse events secondary to anti-programmed death-1 antibodies and preliminary analysis on the impact of corticosteroids on anti-tumour response: A case series. Eur J Cancer. 2018;105:88-102.

29. Kuswanto WF, MacFarlane LA, Gedmintas L, Mulloy A, Choueiri TK, Bermas BL. Rheumatologic symptoms in oncologic patients on PD-1 inhibitors. Semin Arthritis Rheum. 2018;47:907-910. 